\title{
Praehabilitation bei onkologischen Erkrankungen
}

Praehabilitation nützt die prätherapeutische Zeitperiode ab Diagnosestellung, um einer funktionalen Verschlechterung und deren negativen klinischen Konsequenzen - vor allem durch Nebenwirkungen von für das Überleben notwendigen Krebstherapien - vorzubeugen.

$\mathbf{P}$ raehabilitation (auch Prähabilitation oder Prehabilitation) ist jener Teil des sogenannten Cancer-Care-Kontinuums, der neben all den anderen medizinischen Maßnahmen wie Aufklärung, prätherapeutischen diagnostischen Maßnahmen etc. in der Zeit ab der Krebsdiagnose bis zur therapeutischen Intervention stattfindet (siehe Abbildung). Es konnte gezeigt werden, dass die Intervention der Praehabilitation den funktionalen Status sowie den körperlichen und psychischen Outcome signifikant verbessern kann.

Bei der Praehabilitation unterscheidet man den unimodalen vom multimodalen Ansatz. Beim unimodalen Ansatz werden ausschließlich Aspekte der Bewegungstherapie bzw. der Medizinischen Trainingstherapie eingesetzt. Multimodale Ansätze stellen Kombinationen aus Bewegungstherapie, Medizinischer Trainingstherapie, Patient*inneninformation und -schulung, Diätologie und Ernährungstherapie, Psychologie bzw. Psychoonkologie, Raucherentwöhnung und Alkoholreduktion dar. Beide Ansätze zielen darauf ab, die funktionale Kapazität, also die körperliche Kondition und klinische sowie auch die mentale Belastbarkeit der Patient*innen, zu verbessern und diese in einen Zustand zu versetzen, dass sie die klinischen Belastungen der folgenden überlebensnotwendigen Krebsbehandlung leichter überstehen und auch besser damit umgehen können. Die Komplikationsrate kann reduziert werden und Entlassungen

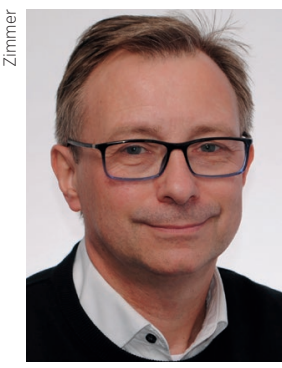

Von

Univ.-Prof. Dr. Richard Crevenna, MBA, MMSC

Vorstand der Universitätsklinik für Physikalische Medizin, Rehabilitation und Arbeitsmedizin, MedUni Wien

können früher erfolgen. So können unter anderem pulmonale Komplikationen bei bzw. nach thorakalen und abdominalen Eingriffen, aber auch das Symptom Inkontinenz nach radikalen Prostatektomien in vielen Fällen deutlich reduziert werden. Für Brustkrebspatientinnen konnte u. a. gezeigt werden, dass die postoperative Beweglichkeit des Schultergürtels nach Praehabilitation deutlich besser sein kann. Auch onkologische Therapien wie Chemotherapien und Strahlentherapien können besser vertragen werden. Bei mentalen Themen wie Angst und Distress scheint die Praehabilitation laut Studienlage eine positive Wirkung zu haben, zusätzlich kann sie dazu führen, dass onkologische Patient*innen leichter motiviert werden können, nach der auf die Praehabilitation folgenden onkologischen Primärbehandlung auch eine onkologische Rehabilitation in Anspruch zu nehmen. Die Patient*innen kommen auch früher in einen entsprechenden Zustand der sogenannten Rehabilitationsfähigkeit, $d$.h. dass für sie zum Beispiel nach schwerwiegenden, großen Eingriffen aufgrund ihres besseren funktionalen Status eine Rehabilitation schon zu einem früheren Zeitraum in Frage kommen kann.

Für die Sinnhaftigkeit der Intervention Praehabilitation gibt es mittlerweile eine zunehmend wachsende wissenschaftliche Evidenz mit guten Belegen der Wirksamkeit bei einzelnen Indikationen. Es besteht allerdings weiterer Forschungsbedarf hinsichtlich Effektivität und Effizienz der Praehabilitation insgesamt: beim unimodalen Ansatz speziell zum Einsatz der Bewegungstherapie bzw. der Medizinischen Trainingstherapie, bei multimodalen Ansätzen für die Wertigkeit der Einzelkomponenten sowie für die Kombination dieser - jeweils für die unterschiedlichen Entitäten und jeweiligen krebs- und stadienspezifischen Primärtherapien, die auf die Praehabilitation folgen.

Wichtig wird es auch sein herauszufinden, wie etwaige Barrieren (u.a. die erforderliche Zeit bzw. Termine für die Prehabilitation in dem oft kurzen Zeitraum zwischen Diagnostik und Beginn der Krebsbehandlung unterzubringen bzw. auch durch Motivation und notwendige Adherence der Patient*innen zu erreichen) überwunden werden können und dabei auch klar zu definieren, für welche Entitäten eine Praehabilitation sinnvoll sein kann und für welche nicht. Sicher ist hier auch der zusätzliche Einsatz telerehabilitativer Ansätze eine vielversprechende Möglichkeit.

Abbildung: Multimodale Praehabilitation und ihre positiven Effekte im Cancer-Care-Kontinuum

\begin{tabular}{|c|c|c|c|c|}
\hline & PRAEHABILITATION & & KREBSTHERAPIE & \\
\hline \multirow[t]{7}{*}{ Krebsdiagnose } & Übungen/Training & Unmittelbare Effekte & Chemotherapie & Effekte im Anschluss an die Therapie \\
\hline & Ernährungstherapie & Funktionaler Status & Strahlentherapie & Kardiorespiratorische Fitness \\
\hline & Psychologische & Kardiorespiratorische & Operative & Muskelkraft \\
\hline & Unterstüzung & Reserve & Eingriffe & Physischer und funktionaler Status \\
\hline & Lifestyle- & & Biologische & Fatigue \\
\hline & Modifikation & & Therapie & Postoperative Komplikationen \\
\hline & & & & Dauer des Spitalsaufenthalts \\
\hline
\end{tabular}

Quelle: Crevenna R et al. Cancer prehabilitation-a short review. MEMO. 2021, https://doi.org/10.1007/s12254-021-00686-5 